\title{
What Is Meaning: Reference or Something Else?
}

\author{
Xinyu Zhao \\ Nanjing University of Science and Technology ZiJin College, Nanjing 210000, Jiangsu, China \\ Email: janeraser@163.com
}

Abstract: The debate over the meaning of referential terms has a long history in both linguistics and language philosophy. Moore applied referential theory of meaning in two famous arguments: The Refutation of Idealism and The Open Question Argument. The validity of referential theory is key point to decide whether these two arguments are successful or not. This paper argues that the plausibility of referential theory is subject to controversy and thus the conclusions of these two arguments are logically invalid. This research firstly demonstrates the logic behind Moore's two arguments and explain how Moore reached his conclusions by assuming the referential theory of meaning, then discusses problems that referentialism inevitably confronts, proving the solutions that Moore proposed to solve these problems are unfeasible, and concludes that these arguments fail for it doesn't comply with our rational intuition, providing linguistic perspective to examine philosophical problems.

Keywords: meaning, reference, G. E. Moore, logic

\section{Introduction}

G. E. Moore's Refutation of Idealism is a well-known attempt at undermining the argument for modern idealism by challenging what is, in his opinion, a necessary and common premise in these arguments, "esse is percipi". Moore is also famous for his Open Question Argument in which he claims that any attempt to identify morality with some set of observable, natural properties will always be liable to an open question, and that if this is true, then moral facts cannot be reduced to natural properties and that therefore ethical naturalism is incorrect. ${ }^{[1]}$ Both of these two arguments rely on referential theory of meaning that argues the meaning of an expression is exhausted by its reference which is the object this expression refers to. In the first argument, the referential theory works as the first premise, while in the second argument, without this assumption, the move from premise two to premise three will be logically false. Therefore, if this theory is implausible, then at least any logical compulsion attached to his arguments will be removed.

\section{The refutation of idealism}

Moore proposed that "there are just three possible meaning, one or other of which such a statement must have, if it is to be true; and of these three is only one which it can have, if it is to be important." ${ }^{22]}$ This research focuses on the first possible meaning that Moore quickly dropped.

The statement may be meant to assert that the word esse is used to signify nothing either more or less than the word percipi: that the two words are precise synonyms: that they are merely different names for one and the same thing: that what is meant by esse is absolutely identical with what is meant by percipi. I think I need not prove that the principle esse is percipi is not thus intended merely to define a word, nor yet that, if it were, it would be an extremely bad definition. ${ }^{[1]}$

Moore thought that the first possible meaning of "esse is percipi" is that "esse" and "percipi" refers to the same thing which means no more than what is referred to by "esse" is absolutely identical to what is referred to by "pericipi". And if the object refered to by "esse" is absolutely the same as that referred to by "percipi", these two terms, "esse" and "percipi", have the same meaning and thus "esse is percipi" expresses a trivial proposition. Moore"s argument can be restructured in this way:

P1 If "A" and "B" refer to the same thing, they have the same meaning.

$\mathrm{P} 2$ If "A and "B" have the same meaning, the proposition expressed by "A is B" is trivial.

P3 Modern idealists will not include a trivial proposition as a premise in their arguments.

C2 If "esse" and "percipi" refer to the same thing, they have the same meaning. 
C2 If "esse and percipi" have the same meaning, the proposition expressed by "esse is percipi" is trivial.

$\mathrm{C} 3$ modern idealists must not intend to mean "esse is percipi" in this way.

It is obvious here that if the assumption of this argument that the meaning of an expression consists only of its reference is proved to be untenable, Moore's attempt to refute idealism cannot be considered as a successful one.

\section{Open question argument}

Consider any suggested naturalistic analysis $\mathrm{N}$ of a moral predicate $\mathrm{M}$. The Open Question Argument maintains that it will always be possible for someone competent with moral discourse without conceptual confusion to grant that something is N but still wonder whether it is really M. ${ }^{[3]}$ In his open question argument, Moore concluded that "GOOD" is unanalyzable and then he extended this conclusion to all moral concepts. Again, this argument is also based on referential theory of meaning - meaning is exhausted by its reference. Moore asserted that the reference of an expression is the object referred to by this expression, while the reference of a sentence is the proposition this sentence expressed. Moore also endorsed the principle of compositionality: the meaning of a complex expression depends on the meanings and arrangements of its constituent parts. ${ }^{[4]}$ In terms of this principle, if a constituent expression A in a sentence doesn't have reference, the sentence lacks reference too. On the other hand, two sentences express different proposition, then the constituent expressions of these two sentences refers to different objects. Moore proposed that if X (X can be any natural terms) is good, then sentence "Is X good" will expresses the same proposition as "Is good good". But because the first question is open while the second one is close, namely obviously true, so they express different propositions. According to principle of compositionality, consequently, "X" and "good" must have different reference. Therefore, they have different meanings. The argument can be demonstrated in this way:

P1 The meaning of a sentence is its reference and the reference of a sentence is the proposition it expresses.

P2 Suppose that A is good means the same as A is what we desire to desire.

P3 Then "is what we desire to desire good?" Is the same question as "is what we desire to desire what we desire to desire?"

P4 But the proposition of first question is significant: there would be a point in asking it, while that of the second question is trivial: it is kind stupid to ask it.

C1 The two questions do not mean the same.

$\mathrm{C} 2 \mathrm{~A}$ is good does not mean $\mathrm{A}$ is something we desire to desire.

P5 Principle of compositionality.

C3 Good is not what we desire to desire.

The premise 3 derived from the premise 2 assumes that if "good" and "what we desire to desire" refer to the same thing, then they have the same meaning.

\section{Logic problems of the referential theory of meaning}

\subsection{Fictional characters}

Although many philosophers have contradicted Moore's arguments from various perspectives, it should be noted that the referential theory of meaning (RTM) is problematic linguistically per se, and can lead to contradiction when being applied to sentences containing fictional characters: individuals whose behavior is written about in works of fiction and who make their first appearance in a work of fiction. ${ }^{[5]}$

It is naturally to assume that if sentence $\mathrm{S}$ is true in $\mathrm{L}$, then $\mathrm{S}$ is meaningful in L. A sentence is true if and only if there is at least one object to which the subject expression refers, and this object has the property expressed by the predicate. ${ }^{[6]}$ If two sentences have the same proposition, they must have the same truth-value. Under the principle of compositionality, if we accept referential theory of meaning, we must admit that if a constituent expression of a sentence lacks reference, namely meaning, this sentence will lack reference, namely meaning, too. Let us consider following argument:

P1 Principle of compositionality.

P2 Sentence "Sherlock Holmes is a detective" is meaningful.

$\mathrm{C} 1$ "Sherlock Holmes" is meaningful.

P3 RTM (the referential theory of meaning): the meaning of an expression is exhausted by the reference of an 
expression.

P4 The reference of "Sherlock Holmes" doesn't exist.

C2 "Sherlock Holmes" has no meaning.

C3 Sentence "Sherlock Holmes is a detective" is meaningless.

In the argument above, since these premises entail the conclusion $\mathrm{C} 3$ which is contradictory to the second premise, at least one of these premises must be false. For principle of compositionality is generally accepted, it is assumed to be right in this paper. And it is intuitively wrong to say premise 2 is false. To every competent language user, "Sherlock Holmes was a detective" is meaningful. It is easy to understand this sentence whether it is written down or spoken. This sentence also can be easily translated into different languages and still makes sense. To say this sentence is meaningless is no less weird than to say sky is yellow. Therefore, the premise 2 is safe. It is plain that premise 4 is true because Sherlock Holmes is just an artificial character created by a novelist. The only way to fix the paradox in the argument above is to reject RTM then.

\subsection{Negative existential}

Negative Existential is another problem for RTM. Because if RTM is true, then it will be impossible to say something have no existing doesn't exist. The Negative Existential argument can be put like this:

P1 Moore: the meaning of an expression consists only of its reference.

P2 A meaningful subject-predicate sentence is true if and only if there is at least one object to which the subject expression refers, and this object has the property expressed by the predicate.

C1 The sentence "Harry Potter doesn't exist" can be true only if there at least one object to which its subject expression refers and these objects have the property of not existing.

P3 If there are objects to which the subjects of meaningful negative existential refer, then they exist. There are no objects have the property of not existing.

P4 Either Harry Potter exists, or it doesn't have the property expressed by the predicate 'does not exist'.

$\mathrm{C} 2$ There is no meaningful negative existential.

Because the reference of 'Harry Potter' doesn't exist, according to RTM, "Harry Potter doesn't exist" cannot be true. However, this is incompatible with out semantic intuition and every rational language user will frown at this way to understand and use our language.

\section{Moore's resolution of problems RTM confronts}

Moore refuted a common premise in these challenging arguments above, which is "if Harry Potter and Sherlock Holmes don't exist, then there are no references for them". Early in his career, Moore proposed that every objects of thought must have some kind of being. "Being comes in degrees, including a category of nonexistent things that have being to a lower degree than do the things that exist." ${ }^{[7]}$ On this view, there really are such things as Harry Potter and Sherlock Holmes; they possess being, even though they do not exist. Being belongs to every conceivable term, namely every possible object of thought. Being belongs to whatever can be counted. ${ }^{[7]}$ If Harry Potter can be discussed, Harry Potter must be something. If Harry Potter were nothing, it could not be said not to be. Moore suggested that there are two different and individually independent worlds of things having being, one world is comprised of things that exist and the other is of things that don't exist. The later one will be referred as "world N" in this paper. Moore argued that there is a referent of Harry Potter for sure, and the referent belongs to world $\mathrm{N}$ in which things are endowed with some "being" less than that of the other world. Therefore, subjects of "Sherlock Holmes is a detective and Harry Potter doesn't exist" have refer to something of some sort of "being" and sentences containing these subjects are meaningful.

\section{Reasons why Moore's resolution is unfeasible}

This "two world" statement sounds like such a brilliant move to fix the problems of RTM; however, it inevitably leads to new puzzles. It is naturally to assume that we can confirm that we know something is true if and only if we have a method to examine the truth of what we know about it. For example, we can confirm that we know "apple is red" by visual perception; we can confirm that we know " $2+2=4$ " by putting two apples and another two apples together and having four apples in hands; we can confirm we know there is a kind of air without color and smell surrounding us by a scientific 
device. However, we cannot prove the truth of any fictional characters produced by J.k.Rowling in any way. The fact that "Harry Potter" is written down by someone is not sufficient to justify the truth of "Harry Potter". Otherwise an individual can automatically become a professor of Harvard University by just writing down that "I am a professor and teach in Harvard" without being admitted by and attending to this university. There is no way to know the truth of things in world $\mathrm{N}$ except that things in world $\mathrm{N}$ doesn't exist. Therefore, even though there is a referent for "Sherlock Holmes" in world $\mathrm{N}$, we still cannot confirm that, in the sentence "Sherlock Holmes is a detective", whether "Sherlock Holmes" satisfies its predicate "is a detective". The truth-value of propositions expressed by sentences containing things from the world $\mathrm{N}$ is inaccessible to us forever. In other words, sentences "Sherlock Holmes is a detective" and "Sherlock Holmes is not a detective" are both of no truth value to us. This fact made Moore's "two worlds" solution incompatible with the Law of Excluded Middle, according to which one of "p" and "not p" must be true.

Moreover, since the only thing we know about objects in world $\mathrm{N}$ is that they have no existing, any attempts trying to differentiate one object from others will be unsuccessful. In no way the reference of Harry Potter can be distinguished from that of Sherlock Holmes. In terms of compositionality, if an individual cannot distinguish the referent of Harry Potter from that of Sherlock Holmes, then he or she is uncertain about whether the sentences "Sherlock Holmes is a detective and Harry Potter is a detective" have different meaning. This doesn't comply with our rational intuition. A competent language user will not say "Harry Potter was a detective". It is obvious that these two sentences mean differently. Moore maybe respond that we can distinguish "Sherlock Holmes" and "Harry Potter" and know that they have different reference, because the former refers to the expression "Sherlock Holmes" and the later refers to the expression "Harry Potter". However, if this is the case, then "Sherlock Holmes/Harry Potter exists" will be true simply because "Sherlock Holmes" and "Harry Potter" refer to two different expressions. Also, if Moore says that because Sherlock Holmes and Harry Potter differ in informational content, they have different meanings. Then, at least, the meaning of an expression should also include the informational content rather than only the reference of an expression. Moore's "two worlds" response seems like ad hoc and fails to resolve the problems of referential theory of meaning discussed above.

\section{Conclusion}

The referential theory which believes meaning of an expression is exhausted by its referent will inevitably lead to some paradoxes and puzzles. It fails to explain, even with Moore's "two worlds" resolution, why fictional characters and sentences containing them are meaningful to competent language users. Thus, it is reasonable to reject this meaning theory and take deeper insights into the mechanism of meaning to find a more inclusive approach to cope with language linguistically and philosophically.

\section{References}

[1] Copp, David. Morality, Normativity, and Society. Oxford: Oxford University Press; 2001.

[2] Moore, G. E. The Refutation of idealism. Mind. 1903; 12(48): 433-453. doi: 10.2307/2248251.

[3] Ridge, M. Moral non-naturalism. Available at: http://plato.stanford.edu/entries/moral-nonnaturalism/\#OpeQueArg [Accessed: 7 December 2015].

[4] Szabó and Gendler, Z. Compositionality. Available at: http://plato.stanford.edu/entries/compositionality/ [Accessed: 7 December 2015].

[5] Oltolini, A. and Kroon, F. Fiction. Available at: http://plato.stanford.edu/entries/fiction/ [Accessed: 16 December 2015].

[6] Glanzberg, M. Truth. Available at: http://plato.stanford.edu/entries/truth/ [Accessed: 7 December 2015].

[7] Soames, S. Philosophical analysis in the twentieth century: The dawn of analysis (V. 1). 2nd edn. Princeton: Princeton University Press; 2005. 